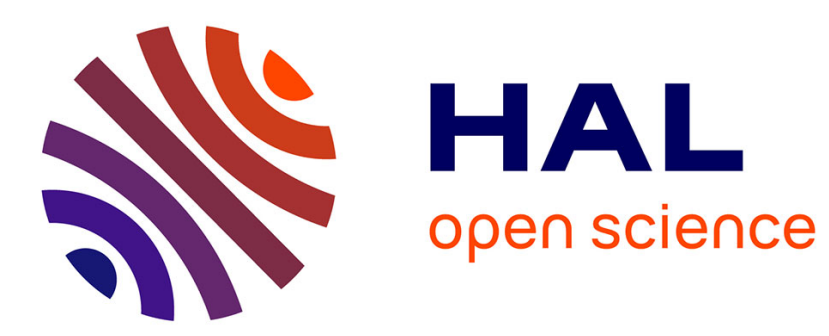

\title{
New cut-balance conditions in networks of clusters
}

Samuel Martin, Irinel-Constantin Morarescu, Dragan Nešic

\section{To cite this version:}

Samuel Martin, Irinel-Constantin Morarescu, Dragan Nešic. New cut-balance conditions in networks of clusters. Automatica, 2017, 77, pp.180-183. 10.1016/j.automatica.2016.11.043 . hal-01580987

\section{HAL Id: hal-01580987 \\ https://hal.science/hal-01580987}

Submitted on 4 Sep 2017

HAL is a multi-disciplinary open access archive for the deposit and dissemination of scientific research documents, whether they are published or not. The documents may come from teaching and research institutions in France or abroad, or from public or private research centers.
L'archive ouverte pluridisciplinaire $\mathbf{H A L}$, est destinée au dépôt et à la diffusion de documents scientifiques de niveau recherche, publiés ou non, émanant des établissements d'enseignement et de recherche français ou étrangers, des laboratoires publics ou privés. 


\title{
New cut-balance conditions in networks of clusters *
}

\author{
Samuel Martin $^{\mathrm{a}} \quad$ Irinel-Constantin Morărescu $^{\mathrm{a}} \quad$ Dragan Nešić $^{\mathrm{b}}$ \\ ${ }^{a}$ Université de Lorraine, CRAN, UMR 7039 and CNRS, CRAN, UMR 7039 \\ 2 Avenue de la Forêt de Haye, Vandouvre-lès-Nancy, France \\ ${ }^{\mathrm{b}}$ Department of Electrical and Electronic Engineering, The University of Melbourne, Parkville, 3010 VIC, Australia.
}

\begin{abstract}
Existing results in the literature guarantee that the state of multi-agent systems interacting over networks that satisfy the cut-balance assumption asymptotically converges to a constant vector. Furthermore, when the network is persistently connected the agents reach a common value called consensus. Many real large-scale networks are obtained by sparsely connecting subnetworks of densely connected agents. In this context, our objective is to provide new cut-balance assumptions that are adapted to networks of clusters. They are useful for consensus and agreement in clusters in situations when network topology is such that clusters are given or can be easily identified. In this case our new cut-balance assumptions can be checked by realizing a smaller number of operations.
\end{abstract}

Key words: Multiagent systems; consensus; structured networks.

\section{Introduction}

The multi-agent framework is widely used to model the dynamics of large numbers of interconnected systems. The most studied problem in this context is the consensus or synchronization of all agents in the network. The convergence to consensus is typically characterized by conditions that depend on the communication graph between agents. Basic results concern fixed undirected topologies but notable advances towards directed and time varying topologies have been provided in (Jadbabaie et al. 2003, Moreau 2005, Ren \& Beard 2005, Hendrickx \& Tsitsiklis 2013) for discrete time dynamics and (Olfati-Saber \& Murray 2004, Ren \& Beard 2005, Hendrickx \& Tsitsiklis 2013, Martin \& Girard 2013, Martin \& Hendrickx 2016) for continuous time algorithms. In (Hendrickx \& Tsitsiklis 2013), the authors introduced the assumption of cut-balance communication which is a general form of communication reciprocity among the agents. Under the cut-balance assumption, convergence is ensured, and consensus may occur in groups or globally. The cut-balance assumption was extended in (Martin \& Girard 2013) where

\footnotetext{
^ This paper was not presented at any IFAC meeting. Corresponding author I.-C. Morărescu.

This work was supported by the ANR project COMPACS"Computation Aware Control Systems", ANR-13-BS03-004 and by the Australian Research Council under the Discovery Project DP1094326.

Email

addresses: samuel.martineuniv-lorraine.fr (Samuel

Martin), constantin.morarescueuniv-lorraine.fr

(Irinel-Constantin

Morărescu), dnesic@unimelb.edu.au (Dragan Nešić).
}

the authors also provided a convergence rate when global consensus takes place. One drawback of the cut-balance assumption is that it is a global assumption which may be hard to verify when not ensured by design.

A direction to search for a local assumption is to split the agents into clusters. It is reported in the literature that large scale networks often consist of sparsely interconnected clusters of densely coupled agents (Chow \& Kokotović 1985, Biyık \& Arcak 2007, Morărescu et al. 2016). Different algorithms have been developed to detect the clusters in such networks (Newman \& Girvan 2004, Blondel et al. 2008, Morărescu \& Girard 2011). In the sequel we take advantage of the partition of network in clusters to state new conditions for consensus.

Consequently, the contribution of the present study is that, under stronger assumptions on the interaction graph, we provide a new assumption on reciprocity of communication which can be verified in a local manner. Therefore, we provide conditions for consensus that can be checked by performing a reduced number of operations.

Notation. The following notation will be used throughout the paper. The set of nonnegative integers, real and nonnegative real numbers are denoted by $\mathbb{N}, \mathbb{R}$ and $\mathbb{R}_{+}$, respectively. A non trivial subset $S$ of a set $C$, denoted as $S \sqsubset C$, is a non-empty set with $S \subsetneq C$.

\section{Problem formulation}

Let $\mathcal{N} \triangleq\{1, \ldots, n\}$ be a set of $n$ agents. By abuse of notation we denote both the agent and its index by the same symbol $i \in \mathcal{N}$. Each agent is characterized by a scalar state $x_{i} \in$ 
$\mathbb{R}, \forall i \in \mathcal{N}$ that evolves according to the following model

$$
\dot{x}_{i}(t)=\sum_{j=1}^{n} a_{i j}(t)\left(x_{j}(t)-x_{i}(t)\right), \forall i \in \mathcal{N}
$$

where $a_{i j}(t) \geq 0$ are measurable functions of time representing the communication weights/interaction strength. Let $x(t)=\left(x_{1}(t), \ldots, x_{n}(t)\right)^{\top} \in \mathbb{R}^{n}$ be the overall state of the network collecting the states of all the agents. It is noteworthy that $x(t), t \in \mathbb{R}^{+}$is uniquely defined by an initial state $x(0)$ and dynamics (1). Indeed, there exists a unique differentiable function of time $x: \mathbb{R}^{+} \rightarrow \mathbb{R}^{n}$ whose components satisfy equation (1) for all $t \in \mathbb{R}^{+}$. We call it the trajectory of the overall system. We say the trajectory asymptotically reaches a consensus when there exists a common agreement value $\alpha \in \mathbb{R}$ such that

$$
\lim _{t \rightarrow+\infty} x_{i}(t)=\alpha, \forall i \in \mathcal{N}
$$

In the sequel, agents are assumed to be partitioned in $m$ nonempty clusters: $\mathcal{C}_{1}, \mathcal{C}_{2}, \ldots, \mathcal{C}_{m} \subset \mathcal{N}$, that are assumed to be given or can be easily identified. For instance clusters may correspond to groups of agents which are spatially close while different clusters are spatially distant. Let us introduce the following supplementary notation: $\mathcal{M} \triangleq\{1, \ldots, m\}$ and $n_{i}$ denotes the cardinality of cluster $\mathcal{C}_{i}$. Without loss of generality, we permute the agents' labels according to the cluster partition so that when $j \in \mathcal{C}_{i}$ and $j^{\prime} \in \mathcal{C}_{i+1}, j<j^{\prime}$.

Definition $1 A$ directed path of length $p$ in a given $d i$ rected graph $\mathcal{G}=(\mathcal{V}, \mathcal{F})$ is a union of directed edges $\bigcup_{k=1}^{p}\left(i_{k}, j_{k}\right)$ such that $i_{k+1}=j_{k}, \forall k \in\{1, \ldots, p-1\}$. The node $j$ is connected with node $i$ in a directed graph $\mathcal{G}=(\mathcal{V}, \mathcal{F})$ if there exists at least a directed path in $\mathcal{G}$ from $i$ to $j$ (i.e. $i_{1}=i$ and $j_{p}=j$ ).

For two subsets of nodes $A, B \subset \mathcal{N}$, the sum of communication weights from $B$ to $A$ is denoted as

$$
w_{A \leftarrow B}(t)=\sum_{i \in A, j \in B} a_{i j}(t)
$$

The cut-balance assumption in (Hendrickx \& Tsitsiklis 2013) can be formulated as follows.

Hypothesis 1 There exists a constant $K \geq 1$ such that for all non trivial subsets $S \sqsubset \mathcal{N}$

$$
w_{S \leftarrow(\mathcal{N} \backslash S)}(t) \leq K \cdot w_{(\mathcal{N} \backslash S) \leftarrow S}(t), \forall t \geq 0 .
$$

This basically means that if a group of agents influences the remaining ones, the former group is also influenced by the remaining ones by at least a proportional amount. A comparison between the cut-balance condition and other types of communication such as existence of a spanning tree has been carried out in (Hendrickx \& Tsitsiklis 2013, Martin \& Girard 2013). Let us recall here the first part of Theorem 1 in (Hendrickx \& Tsitsiklis 2013). First, we define the graph of persistent communication.

Definition 2 A persistent edge associated with system (1) is a couple $(j, i) \in \mathcal{N} \times \mathcal{N}$ such that $\int_{0}^{\infty} a_{i j}(t) d t=+\infty$. The graph of persistent communication associated to system (1) is the graph $G=(\mathcal{N}, \mathcal{E})$ gathering all agents and including only the persistent edges, i.e.,

$$
\mathcal{E}=\left\{(j, i) \in \mathcal{N} \times \mathcal{N} \mid \int_{0}^{\infty} a_{i j}(t) d t=+\infty\right\}
$$

Theorem 1 Suppose that Hypothesis 1 is satisfied for all time $t \geq 0$. Then, the trajectory of system (1) converges. Then, there is a directed path from $i$ to $j$ in the graph of persistent communication $G$ if and only if there is also a directed path from $j$ to $i$, and there holds in that case $\lim _{t \rightarrow \infty} x_{i}(t)=\lim _{t \rightarrow \infty} x_{j}(t)$.

Notice that Hypothesis 1 is a global assumption which may be hard to verify when not ensured by design. The objective of this work is to propose new assumptions that can be verified locally and provides similar guaranties for the particular case of graphs partitioned in clusters. Let us introduce here the main hypotheses of this work.

Assumption 1 (Intra-cluster reciprocity) There exists a constant $K_{I} \geq 1$ such that for any cluster $k \in \mathcal{M}$ and for all non trivial subsets $S \sqsubset \mathcal{C}_{k}$,

$$
w_{S \leftarrow\left(\mathcal{C}_{k} \backslash S\right)}(t) \leq K_{I} \cdot w_{\left(\mathcal{C}_{k} \backslash S\right) \leftarrow S}(t), \forall t \geq 0 .
$$

Assumption 2 (Inter-cluster reciprocity) There exists a constant $K_{E} \geq 1$ such that for all non trivial subset $S \sqsubset \mathcal{M}$,

$$
\sum_{k \in S} w_{\mathcal{C}_{k} \leftarrow\left(\mathcal{N} \backslash \mathcal{C}_{k}\right)}(t) \leq K_{E} \cdot \sum_{k \in S} w_{\left(\mathcal{N} \backslash \mathcal{C}_{k}\right) \leftarrow \mathcal{C}_{k}}(t), \forall t \geq 0
$$

Assumptions 1 and 2 correspond to Hypothesis 1 within each cluster and between clusters, respectively. In Assumption 2 the equivalent cut-balance assumption is formulated in the case where each cluster is considered as a node and the communication between clusters is weighted by the sum of agent-wise communication weights. It is necessary that $K_{I} \geq 1$ and $K_{E} \geq 1$ and the equality corresponds to symmetric communications. The next assumption ensures that the total communication weight which a cluster $\mathcal{C}_{k}$ receives cannot exceed a proportion of the weight received by any non trivial subset of $\mathcal{C}_{k}$ from the rest of $\mathcal{C}_{k}$.

Assumption 3 (Clustered communication) There exists a constant $\rho>0$ such that for each cluster $k \in\{1, \ldots, m\}$ and for all non trivial subsets $S \sqsubset \mathcal{C}_{k}$,

$$
w_{\mathcal{C}_{k} \leftarrow\left(\mathcal{N} \backslash \mathcal{C}_{k}\right)}(t) \leq \rho \cdot w_{S \leftarrow\left(\mathcal{C}_{k} \backslash S\right)}(t), \forall t \geq 0 .
$$

Remark 1 The purpose of Assumption 3 is to prevent cases where two subsets of a cluster are more connected to the outside than to each other. To understand the importance of Assumption 3, we have the two following facts : 
- Assumption 3 is not necessarily satisfied when Assumptions 1 and 2 hold.

- Assumptions 1 and 2 without Assumption 3 are not sufficient to obtain the global cut-balance Hypothesis 1.

A counter-example illustrated by Figure 1 allows to prove these facts : consider the 4-agent system with communications described by $a_{12}(t)=a_{21}(t)=a_{34}(t)=a_{43}(t)=1$ and $a_{13}(t)=a_{42}(t)=t$. All these weights form persistent edges. The other weights are assumed to be uniformly 0 . The only non-trivial partition in clusters satisfying Assumptions 1 and 2 is $C_{1}=\{1,2\}$ and $C_{2}=\{3,4\}$ with $K_{I}=1=K_{E}$. For this partition, Assumption 3 clearly fails for instance taking $S=\{1\}$. Moreover, the global cut-balance Hypothesis 1 also fails for instance taking $S:=\{1,2,4\}$ (see Figure 1 for an illustration). The relation (2) in Hypothesis 1 holds only if $K \geq t, \forall t \geq 0$ i.e., $K=\infty$, which is not feasible.

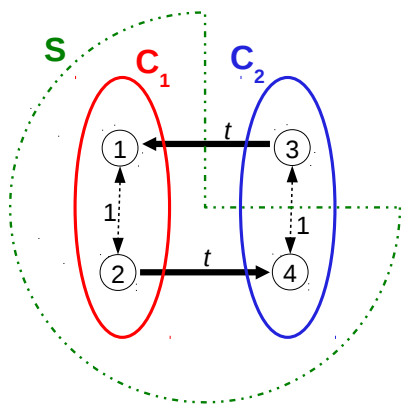

Fig. 1. Illustration of the importance of Assumption 3. Continuous blue and red lines represents the clusters $\mathcal{C}_{1}$ and $\mathcal{C}_{2}$ as in Remark 1. Bold unidirectional arrows correspond to the stronger communication weights with value $t$ while dashed bidirectional arrows correspond to the weaker communcation weights with value 1 . The dotted-dashed green line corresponds to a particular set $S$ which makes the cut-balance Hypothesis 1 fail.

\section{Asymptotic behavior: Consensus and Clustering}

In this section, we suppose that we deal with a network partitioned in clusters satisfying the communication pattern introduced by Assumptions 1, 2 and 3. Our main result can be stated as follows.

Proposition 1 Under Assumptions 1, 2 and 3, the communication weights satisfy Hypothesis 1 with reciprocity constant $K=\left(K_{I}+\rho+K_{E} \max (\rho, 1)\right)$.

Notice that the global cut-balance Hypothesis 1 does not guarantee the existance of a non-trivial partition into clusters for which Assumptions 1, 2 and 3 are all satisfied. For a counter-example, consider the 3-node unidirectional circle graph with constant weights such that $a_{12}(t)=a_{23}(t)=a_{31}(t)=1$ and the other weights are uniformly 0 . The global cut-balanced condition is satisfied but no non-trivial partition into clusters satisfies Assumption 1.

PROOF. Let $S$ be some non trivial subset of $\mathcal{N}$. Denote, for $k \in\{1, \ldots, m\}, S_{k}=S \cap \mathcal{C}_{k}$. Set $S_{k}$ may either be empty, equal to $\mathcal{C}_{k}$ or a non trivial subset of $\mathcal{C}_{k}$. We introduce

$$
\begin{aligned}
& E_{\emptyset}=\left\{k \in\{1, \ldots, m\} \mid S_{k}=\emptyset\right\}, \\
& E_{e q}=\left\{k \in\{1, \ldots, m\} \mid S_{k}=\mathcal{C}_{k}\right\}, \\
& E_{\sqsubset}=\left\{k \in\{1, \ldots, m\} \mid S_{k} \sqsubset \mathcal{C}_{k}\right\} .
\end{aligned}
$$

We have

$$
\begin{aligned}
w_{S \leftarrow(\mathcal{N} \backslash S)}(t) & =\sum_{k=1}^{m} w_{S_{k} \leftarrow(\mathcal{N} \backslash S)}(t) \\
=\sum_{k \in E_{e q}} & w_{S_{k} \leftarrow(\mathcal{N} \backslash S)}(t)+\sum_{k \in E_{\sqsubset}}^{m} w_{S_{k} \leftarrow(\mathcal{N} \backslash S)}(t) .
\end{aligned}
$$

Denote $W_{e q}(t)$ and $W_{\sqsubset}(t)$ the terms in the right-hand side above. First, quantity $\bar{W}_{\sqsubset}(t)$ can be rewritten as

$$
W_{\sqsubset}(t)=\sum_{k \in E_{\sqsubset}} w_{S_{k} \leftarrow\left(\mathcal{C}_{k} \backslash S_{k}\right)}(t)+\sum_{k \in E_{\sqsubset}} w_{S_{k} \leftarrow \mathcal{N} \backslash\left(\mathcal{C}_{k} \cup S\right)}(t) .
$$

Set $S_{k}$ being non-empty, the first term can be upper bounded using Assumption 1. Set $\left(\mathcal{C}_{k} \backslash S_{k}\right)$ being non-empty either, the second term can be upper bounded using Assumption 3 . This yields

$$
\begin{aligned}
W_{\sqsubset}(t) & \leq \sum_{k \in E_{\sqsubset}} K_{I} w_{\left(\mathcal{C}_{k} \backslash S_{k}\right) \leftarrow S_{k}}(t)+\sum_{k \in E_{\sqsubset}} \rho w_{\left(\mathcal{C}_{k} \backslash S_{k}\right) \leftarrow S_{k}}(t) \\
& =\left(K_{I}+\rho\right) \sum_{k \in E_{\sqsubset}} w_{\left(\mathcal{C}_{k} \backslash S_{k}\right) \leftarrow S_{k}}(t) .
\end{aligned}
$$

Secondly, using the definition of $E_{e q}$ we rewrite the quantity $W_{e q}$ as

$$
\begin{aligned}
W_{e q}(t) & =\sum_{k \in E_{e q}} w_{\mathcal{C}_{k} \leftarrow(\mathcal{N} \backslash S)}(t) \\
& \leq \sum_{k \in E_{e q}} \sum_{h \in \mathcal{M} \backslash E_{e q}} w_{\mathcal{C}_{k}} \leftarrow \mathcal{C}_{h}(t),
\end{aligned}
$$

where the last inequality comes from $(\mathcal{N} \backslash S) \subseteq$ $\mathcal{N} \backslash \cup_{k \in E_{e q}} \mathcal{C}_{k}$, so that Assumption 2 applies and gives

$$
\begin{aligned}
& W_{e q}(t) \leq K_{E} \sum_{h \in \mathcal{M} \backslash E_{e q}} \sum_{k \in E_{e q}} w_{\mathcal{C}_{h} \leftarrow \mathcal{C}_{k}}(t) \leq \\
& K_{E}\left(\sum_{h \in E_{\emptyset}} \sum_{k \in E_{e q}} w_{\mathcal{C}_{h} \leftarrow \mathcal{C}_{k}}(t)+\sum_{h \in E_{\sqsubset}} \sum_{k \in E_{e q}} w_{\mathcal{C}_{h} \leftarrow \mathcal{C}_{k}}(t)\right) .
\end{aligned}
$$

The first term in the bracket can be bounded by $\sum_{h \in E_{\emptyset}} w_{\mathcal{C}_{h} \leftarrow S}$ and for the second term we use $E_{\sqsubset} \cap E_{e q}=\emptyset$ to get,

$\sum_{h \in E_{\sqsubset}} \sum_{k \in E_{\text {eq }}} w_{\mathcal{C}_{h} \leftarrow \mathcal{C}_{k}}(t) \leq \sum_{h \in E_{\sqsubset}} w_{\mathcal{C}_{k}} \leftarrow\left(\mathcal{N} \backslash \mathcal{C}_{h}\right)(t)$. 
Since for $k \in E_{\sqsubset},\left(\mathcal{C}_{k} \backslash S_{k}\right)$ is a non trivial subset of $\mathcal{C}_{k}$, we apply Assumption 3 to obtain

$$
\begin{aligned}
\sum_{h \in E_{\sqsubset}} \sum_{k \in E_{e q}} w_{\mathcal{C}_{h} \leftarrow \mathcal{C}_{k}}(t) & \leq \rho \sum_{h \in E_{\sqsubset}} w_{\left(\mathcal{C}_{h} \backslash S_{h}\right) \leftarrow S_{h}}(t) \\
& \leq \rho \sum_{h \in E_{\sqsubset}} w_{\left(\mathcal{C}_{h} \backslash S_{h}\right) \leftarrow S}(t) .
\end{aligned}
$$

Consequently,

$W_{e q}(t) \leq K_{E} \max (\rho, 1) \sum_{k \in E_{\sqsubset} \cup E_{\emptyset}} w_{\left(\mathcal{C}_{k} \backslash S_{k}\right) \leftarrow S}(t)$.

Since $w_{S \leftarrow(\mathcal{N} \backslash S)}(t)=W_{\sqsubset}(t)+W_{e q}(t)$, it holds

$w_{S \leftarrow(\mathcal{N} \backslash S)}(t) \leq\left(K_{I}+\rho+K_{E} \max (\rho, 1)\right) w_{(\mathcal{N} \backslash S) \leftarrow S}(t)$.

In the cases where the clusters are already given, checking Proposition 1 requires a smaller number of operations. Precisely, in the general case, the cut-balance assumption requires to loop over $2^{n}$ sets $S$ and for each $S$ to sum over $O\left(n^{2}\right)$ terms. On the other hand Assumptions 1 and 3 require at most $\max _{k \in\{1, \ldots, m\}} n_{k} 2^{n_{k}}$ such sets with a sum over $O\left(n_{k}^{2}\right)$ terms and Assumption 2 requires a loop over $2^{m}$ sets and sum over $O\left(\mathrm{~m}^{2}\right)$ terms. From Theorem 1, we have the following corollary.

Corollary 1 Suppose that Assumptions 1, 2 and 3 are satisfied. Then, the trajectory of system (1) converges. Let $G=(\mathcal{N}, \mathcal{E})$ be the graph of persistent communication (see Definition 2). Then,

- there is a directed path from $i$ to $j$ in $G$ if and only if there is also a directed path from $j$ to $i$, and there holds in that case $\lim _{t \rightarrow \infty} x_{i}(t)=\lim _{t \rightarrow \infty} x_{j}(t)$,

- if a persistent edge exists between two clusters $\mathcal{C}_{k}, \mathcal{C}_{h}, k \neq$ $h \in \mathcal{M}$, then

$$
\lim _{t \rightarrow \infty} x_{i}(t)=\lim _{t \rightarrow \infty} x_{j}(t), \forall i, j \in \mathcal{C}_{k} \cup \mathcal{C}_{h} .
$$

PROOF. The first item of the corollary is implied by Theorem 1 and Proposition 1. For the second, notice that if there exists a persistent edge $(j, i)$ linking two clusters i.e., with $i \in \mathcal{C}_{k}, j \in \mathcal{C}_{h}$ and $k \neq h$, Assumption 3 implies that the persistent edges internal to cluster $\mathcal{C}_{k}$ form a strongly connected graph, and by the reciprocity Assumption 2, there also exist persistent edges from cluster $\mathcal{C}_{k}$ to $\mathcal{C}_{h}$, so that the persistent edges internal to cluster $\mathcal{C}_{h}$ also form a strongly connected graph. As a conclusion, all pairs of nodes in $\mathcal{C}_{k} \cup \mathcal{C}_{h}$ are linked by a path of persistent edges and the first bullet point applies.

When any two agents belonging to a cluster $\mathcal{C}_{k}, k \in \mathcal{M}$ are connected by a persistent directed path, the first item of
Corollary 1 guarantees that a local agreement is reached in $\mathcal{C}_{k}$. If the local agreements $\alpha_{k}, \alpha_{h} \in \mathbb{R}$ are reached in clusters $\mathcal{C}_{k}, \mathcal{C}_{h}, k \neq h \in \mathcal{M}$, respectively, and a persistent link exists between the two clusters, the second item of Corollary 1 implies $\alpha_{k}=\alpha_{h}$. In other words we provide a flexible characterization of the asymptotic behavior describing agreement in: clusters, groups of clusters or overall network.

\section{Conclusion}

In this note we investigated consensus in network structured in clusters. Our main assumptions guaranteeing consensus in this framework are adaptations of the global cut-balance assumption. We believe that the conditions we propose are better adapted to the clustered communications case because they are local and consequently their verification requires a smaller number of operations.

\section{References}

Biyık, E. \& Arcak, M. (2007), 'Area aggregation and time-scale modeling for sparse nonlinear networks', Systems \& Control Letters 57(2), 142-149.

Blondel, V., Guillaume, J.-L., Lambiotte, R. \& Lefebvre, E. (2008), 'Fast unfolding of communites in large networks', Journal of Statistical Mechanics: Theory and Experiment P10008(10).

Chow, J. \& Kokotović, P. (1985), 'Time scale modeling of sparse dynamic networks', IEEE Transactions on Automatic Control, 30(8), 714-722.

Hendrickx, J. M. \& Tsitsiklis, J. N. (2013), 'Convergence of typesymmetric and cut-balanced consensus seeking systems.', IEEE Transactions on Automatic Control, 58(1), 214-218.

Jadbabaie, A., Lin, J. \& Morse, A. S. (2003), 'Coordination of groups of mobile autonomous agents using nearest neighbor rules', IEEE Transactions on Automatic Control, 48(6), 988-1001.

Martin, S. \& Girard, A. (2013), 'Continuous-time consensus under persistent connectivity and slow divergence of reciprocal interaction weights.', SIAM Journal on Control and Optimization, 51(3), 25682584.

Martin, S. \& Hendrickx, J. (2016), 'Continuous-time consensus under non-instantaneous reciprocity', IEEE Transactions on Automatic Control, DOI: 10.1109/TAC.2015.2506262

Moreau, L. (2005), 'Stability of multiagent systems with time-dependent communication links', IEEE Transactions on Automatic Control, 50(2), 169-182.

Morărescu, I.-C. \& Girard, A. (2011), 'Opinion dynamics with decaying confidence: Application to community detection in graphs', IEEE Transactions on Automatic Control, 56(8), 1862 - 1873.

Morărescu, I.-C., Martin, S., Girard, A. \& Muller-Gueudin, A. (2016), 'Coordination in networks of linear impulsive agents', IEEE Transactions on Automatic Control, DOI: 10.1109/TAC.2015.2492058.

Newman, M. E. J. \& Girvan, M. (2004), 'Finding and evaluating community structure in networks', Phys. Rev. E 69: 026113.

Olfati-Saber, R. \& Murray, R. (2004), 'Consensus problems in networks of agents with switching topology and time-delays', IEEE Transactions on Automatic Control, 49, 1520-1533.

Ren, W. \& Beard, R. W. (2005), 'Consensus seeking in multiagent systems under dynamically changing interaction topologies', IEEE Transactions on Automatic Control, 50(5), 655-661. 